\title{
SPECTRAL-COLLOCATION METHOD FOR FRACTIONAL FREDHOLM INTEGRO-DIFFERENTIAL EQUATIONS
}

\author{
Yin Yang, Yanping Chen, and Yunging Huang
}

\begin{abstract}
We propose and analyze a spectral Jacobi-collocation approximation for fractional order integro-differential equations of FredholmVolterra type. The fractional derivative is described in the Caputo sense. We provide a rigorous error analysis for the collection method, which shows that the errors of the approximate solution decay exponentially in $L^{\infty}$ norm and weighted $L^{2}$-norm. The numerical examples are given to illustrate the theoretical results.
\end{abstract}

\section{Introduction}

Many phenomena in engineering, physics, chemistry, and other sciences can be described very successfully by models using mathematical tools from fractional calculus, i.e., the theory of derivatives and integrals of fractional noninteger order. This allows one to describe physical phenomena more accurately. Moreover, fractional calculus is applied to model the frequency dependent damping behavior of many viscoelastic materials, economics and dynamics of interfaces between nanoparticles and substrates. Recently, several numerical methods to solve fractional differential equations (FDEs) and fractional integro-differential equations (FIDEs) have been proposed.

In this article, we are concerned with the numerical study of the following fractional Fredholm integro-differential equation:

$$
\begin{aligned}
& \begin{array}{l}
D^{\gamma} y(t)=y(t)+\int_{0}^{t} k_{1}(t, \tau) y(\tau) d \tau+\int_{0}^{T} k_{2}(t, \varsigma) y(\varsigma) d \varsigma+f(t), \\
0<\gamma<1, \quad t \in[0, T],
\end{array} \\
& \begin{array}{l}
y(0)=y_{0},
\end{array}
\end{aligned}
$$

Received May 20, 2013; Revised September 11, 2013.

2010 Mathematics Subject Classification. Primary 65R20, 45J05, 65N12.

Key words and phrases. spectral Jacobi-collocation method, fractional order Fredholm integro-differential equations, Caputo derivative.

This work was supported by NSFC Project 11301446, 11271145, China Postdoctoral Science Foundation Grant 2013M531789, Program for Changjiang Scholars and Innovative Research Team in University IRT1179, Project of Scientific Research Fund of Hunan Provincial Science and Technology Department 2013RS4057 and the Research Foundation of Hunan Provincial Education Department 13B116. 
where the source function $f$ and the kernel function $k_{1}, k_{2}$ are given, the function $y(t)$ is the unknown one and $y_{0} \in R$. Here, the given functions $f, k_{1}, k_{2}$ are assumed to be sufficiently smooth on their respective domains $I$ and $0 \leq \tau \leq t \leq T$. In the equation (1), $D^{\gamma}$ denotes the fractional derivative of order $\gamma$ defined as a Caputo derivative.

Differential and integral equations involving derivatives of non-integer order have shown to be adequate models for various phenomena arising in damping laws, diffusion processes, models of earthquake [15], fluid-dynamics traffic model [16], mathematical physics and engineering [29], fluid and continuum mechanics [25], chemistry, acoustics and psychology [2].

Let $\Gamma(\cdot)$ denote the Gamma function. For any positive integer $n$ and $n-1<$ $\gamma<n$, the Caputo derivative $D^{\gamma} f(t)$ is defined as follows:

$$
D^{\gamma} f(t)=\frac{1}{\Gamma(n-\gamma)} \int_{a}^{t} \frac{f^{(n)}(\tau)}{(t-\tau)^{(\gamma-n+1)}} d \tau, \quad t \in[a, b] .
$$

Also, the Riemann-Liouville fractional integral $I^{\gamma}$ of order $\gamma$ is defined as

$$
I^{\gamma} f(t)=\frac{1}{\Gamma(\gamma)} \int_{a}^{t}(t-\tau)^{\gamma-1} f(\tau) d \tau
$$

We note that

$$
I^{\gamma}\left(D^{\gamma} f(t)\right)=f(t)-\sum_{k=0}^{n-1} f^{(k)}(a) \frac{t^{k}}{k !} .
$$

From (4), the fractional integro-differential equation (1) can be described as

$$
\begin{gathered}
D^{\gamma} y(t)=y(t)+\int_{0}^{t} k_{1}(t, \tau) y(\tau) d \tau+\int_{0}^{T} k_{2}(t, \varsigma) y(\varsigma) d \varsigma+f(t), \\
0<\gamma<1, \quad t \in[0, T], \\
y(t)=\frac{1}{\Gamma(\gamma)} \int_{0}^{t}(t-\tau)^{\gamma-1} D^{\gamma} y(\tau) d \tau+y(0) .
\end{gathered}
$$

Several methods have been introduced to solve FDEs in analytical and numerical frames. Analytical methods include various transformation techniques [18], operational calculus methods [24], the Adomian decomposition method [38], and the iterative and series-based method [37]. A small number of algorithms for the numerical solution of FDEs have been suggested [1], and most of them are finite difference methods, which are generally limited to low dimensions and are of limited accuracy.

As we know, fractional derivatives are global (they are defined by an integral over the whole interval $[0, T])$, and therefore global methods such as spectral methods are perhaps better suited for FDEs. Standard spectral methods possess an infinite order of accuracy for the equations with regular solutions, while failing for many complicated problems with singular solutions. So, it is relevant to be interested in how to enlarge the adaptability of spectral methods, and 
construct certain simple approximation schemes without a loss of accuracy for more complicated problems.

Spectral methods have been proposed to solve fractional differential equations, such as the Legendre collocation method [20, 36], Legendre wavelets method [32, 34], homotopy perturbation method [40] and Jacobi-Gauss-Lobatto collocation method [4]. The authors in [12, 13, 39] constructed an efficient spectral method for the numerical approximation of fractional integro-differential equations based on tau and pseudo-spectral methods. Moreover, Bhrawy et al. [7] introduced a quadrature shifted Legendre tau method based on the Gauss-Lobatto interpolation for solving multi-order FDEs with variable coefficients and in [6], shifted Legendre spectral methods have been developed for solving fractional-order multi-point boundary value problems. In [35], truncated Legendre series together with the Legendre operational matrix of fractional derivatives are used for the numerical integration of fractional differential equations. In [8] the authors derived a new explicit formula for the integral of shifted Chebyshev polynomials of any degree for any fractional-order. The shifted Chebyshev operational matrix [5] and shifted Jacobi operational matrix [14] of fractional derivatives have been developed, which are applied together with the spectral tau method for numerical solution of general linear multi-term fractional differential equations. However, very few theoretical results were provided to justify the high accuracy numerically obtained. Recently, Chen and Tang $[10,41]$ developed a novel spectral spectral Jacobi-collocation method to solve second kind Volterra integral equations with a weakly singular kernel and provided a rigorous error analysis which theoretically justifies the spectral rate of convergence. Inspired by the work of [41], we extend the approach to fractional order integro-differential equations and provide a rigorous convergence analysis for the Jacobi-collocation method, which indicates that the proposed method converges exponentially provided that the data in the given FIDE are smooth.

This paper is organized as follows. In Section 2, we outline the spectral approach for (1). Some lemmas useful for establishing the convergence result will be provided in Section 3. The convergence analysis will be carried out in Section 4, and Section 5 contains numerical results, which will be used to verify the theoretical result obtained in Section 4.

\section{Jacobi-collocation method}

Let $\omega^{\alpha, \beta}(x)=(1-x)^{\alpha}(1+x)^{\beta}$ be a weight function in the usual sense for $\alpha, \beta>-1$. The set of Jacobi polynomials $\left\{J_{n}^{\alpha, \beta}(x)\right\}_{n=0}^{\infty}$ forms a complete $L_{\omega^{\alpha, \beta}}^{2}(-1,1)$-orthogonal system, where $L_{\omega^{\alpha, \beta}}^{2}(-1,1)$ is a weighted space defined by

$$
L_{\omega^{\alpha, \beta}}^{2}(-1,1)=\left\{v: v \text { is measurable and }\|v\|_{\omega^{\alpha, \beta}}<\infty\right\}
$$


equipped with the norm

$$
\|v\|_{\omega^{\alpha, \beta}}=\left(\int_{-1}^{1}|v(x)|^{2} \omega^{\alpha, \beta}(x) d x\right)^{\frac{1}{2}},
$$

and the inner product

$$
(u, v)_{\omega^{\alpha, \beta}}=\int_{-1}^{1} u(x) v(x) \omega^{\alpha, \beta}(x) d x \quad \forall u, v \in L_{\omega^{\alpha, \beta}}^{2}(-1,1) .
$$

For a given $N \geq 0$, we denote by $\left\{\theta_{k}\right\}_{k=0}^{N}$ the Legendre points, and by $\left\{\omega_{k}\right\}_{k=0}^{N}$ the corresponding Legendre weights (i.e., Jacobi weights $\left\{\omega_{k}^{0,0}\right\}_{k=0}^{N}$ ). Then, the Legendre-Gauss integration formula is

$$
\int_{-1}^{1} f(x) d x \approx \sum_{k=0}^{N} f\left(\theta_{k}\right) \omega_{k}
$$

where $\omega_{k}=\omega^{0,0}\left(x_{k}\right)$. Similarly, we denote by $\left\{\tilde{\theta}_{k}\right\}_{k=0}^{N}$ the Jacobi-Gauss points, and by $\left\{\omega_{k}^{\alpha, \beta}\right\}_{k=0}^{N}$ the corresponding Jacobi weights. Then, the Jacobi-Gauss integration formula is

$$
\int_{-1}^{1} f(x) \omega^{\alpha, \beta}(x) d x \approx \sum_{k=0}^{N} f\left(\tilde{\theta}_{k}\right) \omega_{k}^{\alpha, \beta},
$$

where $\omega_{k}^{\alpha, \beta}=\omega^{\alpha, \beta}\left(x_{k}\right)$.

For a given positive integer $N$, we denote the collocation points by $\left\{x_{i}^{\alpha, \beta}\right\}_{i=0}^{N}$, which is the set of $(N+1)$ Jacobi-Gauss points, corresponding to the weight $\omega^{\alpha, \beta}(x)$. Let $\mathcal{P}_{N}$ denote the space of all polynomials of degree not exceeding $N$. For any $v \in C[-1,1]$, we can define the Lagrange interpolating polynomial $I_{N}^{\alpha, \beta} v \in \mathcal{P}_{N}$, satisfying

$$
I_{N}^{\alpha, \beta} v\left(x_{i}^{\alpha, \beta}\right)=v\left(x_{i}^{\alpha, \beta}\right), \quad 0 \leq i \leq N .
$$

The Lagrange interpolating polynomial can be written in the form

$$
I_{N}^{\alpha, \beta} v(x)=\sum_{i-0}^{N} v\left(x_{i}^{\alpha, \beta}\right) F_{i}(x), \quad 0 \leq i \leq N,
$$

where $F_{i}(x)$ is the Lagrange interpolation basis function associated with

$$
\left\{x_{i}^{\alpha, \beta}\right\}_{i=0}^{N} \text {. }
$$

For the sake of applying the theory of orthogonal polynomials, we use the change of variables to transfer the integration interval $[0, T]$ to a fixed interval $I=:[-1,1]$,

$$
\begin{aligned}
t & =\frac{1}{2} T(1+x), & x & =\frac{2 t}{T}-1, \\
\tau & =\frac{1}{2} T(1+s), & s & =\frac{2 \tau}{T}-1,
\end{aligned}
$$


and let

$$
\varsigma=\frac{1}{2} T(1+\xi), \quad \xi=\frac{2 \varsigma}{T}-1
$$

$u(x)=y\left(\frac{1}{2} T(1+x)\right), D^{\gamma} u(x)=D^{\gamma} y\left(\frac{1}{2} T(1+x)\right), g(x)=f\left(\frac{1}{2} T(1+x)\right)$,
$K_{1}(x, s)=\frac{T}{2} k_{1}\left(\frac{1}{2} T(1+x), \frac{1}{2} T(1+s)\right), K_{2}(x, \xi)=\frac{T}{2} k_{2}\left(\frac{1}{2} T(1+x), \frac{1}{2} T(1+\xi)\right)$.

The fractional integro-differential equation in one dimension (1) or (5) is of the form

$$
\begin{aligned}
& D^{\gamma} u(x)=u(x)+\int_{-1}^{x} K_{1}(x, s) u(s) d s+\int_{-1}^{1} K_{2}(x, \xi) u(\xi) d \xi+g(x), \\
& \quad 0<\gamma<1, \quad x \in I, \\
& u(x)=\frac{1}{\Gamma(\gamma)}\left(\frac{T}{2}\right)^{\gamma} \int_{-1}^{x}(x-s)^{\gamma-1} D^{\gamma} u(s) d s+u(-1) .
\end{aligned}
$$

Let $-\mu=\gamma-1$. Set the collocation points $\left\{x_{i}^{-\mu,-\mu}\right\}_{i=0}^{N}$ as the set of $(N+1)$ Jacobi-Gauss points associated with $\omega^{-\mu,-\mu}(x)$. Assume that Eq. (8) holds at $x_{i}^{-\mu,-\mu}$ :

$$
\begin{aligned}
& D^{\gamma} u\left(x_{i}^{-\mu,-\mu}\right)=u\left(x_{i}^{-\mu,-\mu}\right)+\int_{-1}^{x_{i}^{-\mu,-\mu}} K_{1}\left(x_{i}^{-\mu,-\mu}, s\right) u(s) d s \\
& +\int_{-1}^{1} K_{2}\left(x_{i}^{-\mu,-\mu}, \xi\right) u(\xi) d \xi+g\left(x_{i}^{-\mu,-\mu}\right), \\
& \text { (9b) } u\left(x_{i}^{-\mu,-\mu}\right)=\frac{1}{\Gamma(\gamma)}\left(\frac{T}{2}\right)^{\gamma} \int_{-1}^{x_{i}^{-\mu,-\mu}}\left(x_{i}^{-\mu,-\mu}-s\right)^{-\mu} D^{\gamma} u(s) d s+u(-1) \text {. }
\end{aligned}
$$

The main difficulty in obtaining a high order of accuracy is to compute the integral term in (9). In particular, for small values of $x_{i}^{-\mu,-\mu}$, there is little information available for $u(s)$. To overcome this difficulty, we will transfer the integration interval $\left[-1, x_{i}^{-\mu,-\mu}\right]$ for a fixed $i$ to a fixed interval $[-1,1]$, and then make use of some appropriate quadrature rule. More precisely, we first make a simple linear transformation:

$$
s(x, \theta)=\frac{1+x}{2} \theta+\frac{x-1}{2}, \quad-1 \leq \theta \leq 1 .
$$

Then (9) becomes

$$
\begin{aligned}
& D^{\gamma} u\left(x_{i}^{-\mu,-\mu}\right) \\
= & u\left(x_{i}^{-\mu,-\mu}\right) \\
& +\frac{1+x_{i}^{-\mu,-\mu}}{2} \int_{-1}^{1} K_{1}\left(x_{i}^{-\mu,-\mu}, s\left(x_{i}^{-\mu,-\mu}, \theta\right)\right) u\left(s\left(x_{i}^{-\mu,-\mu}, \theta\right)\right) d \theta \\
& +\int_{-1}^{1} K_{2}\left(x_{i}^{-\mu,-\mu}, \xi\right) u(\xi) d \xi+g\left(x_{i}^{-\mu,-\mu}\right),
\end{aligned}
$$




$$
\text { (11b) } \begin{aligned}
& u\left(x_{i}^{-\mu,-\mu}\right) \\
= & \frac{1}{\Gamma(\gamma)}\left(\frac{T\left(1+x_{i}^{-\mu,-\mu}\right)}{4}\right)^{\gamma} \int_{-1}^{1}(1-\theta)^{-\mu} D^{\gamma} u\left(s\left(x_{i}^{-\mu,-\mu}, \theta\right)\right) d s+u(-1) .
\end{aligned}
$$

Next, using a $(N+1)$-point Gauss quadrature formula relative to the Legendre weights $\left\{\omega_{k}\right\}_{k=0}^{N}$ (i.e., Jacobi weights $\left\{\omega_{k}^{0,0}\right\}_{k=0}^{N}$ ), the integration term in (11a) can be approximated by

$$
\begin{gathered}
\int_{-1}^{1} K_{1}\left(x_{i}^{-\mu,-\mu}, s\left(x_{i}^{-\mu,-\mu}, \theta\right)\right) u\left(s\left(x_{i}^{-\mu,-\mu}, \theta\right)\right) d \theta \\
\approx \sum_{k=0}^{N} K_{1}\left(x_{i}^{-\mu,-\mu}, s\left(x_{i}^{-\mu,-\mu}, \theta_{k}\right)\right) u\left(s\left(x_{i}^{-\mu,-\mu}, \theta_{k}\right)\right) \omega_{k}^{0,0} \\
\int_{-1}^{1} K_{2}\left(x_{i}^{-\mu,-\mu}, \xi\right) u(\xi) d \xi \approx \sum_{k=0}^{N} K_{2}\left(x_{i}^{-\mu,-\mu}, \theta_{k}\right) u\left(\theta_{k}\right) \omega_{k}^{0,0}
\end{gathered}
$$

where $\left\{\theta_{k}\right\}_{k=0}^{N}$ is the set of Jacobi-Gauss points corresponding to the set of Jacobi weights $\left\{\omega_{k}^{0,0}\right\}_{k=0}^{N}$, i.e., $\left\{\theta_{k}\right\}_{k=0}^{N}$ is Legendre-Gauss points.

$$
\int_{-1}^{1}(1-\theta)^{\mu} D^{\gamma} u\left(s\left(x_{i}^{-\mu,-\mu}, \tilde{\theta}\right)\right) d s \approx \sum_{k=0}^{N} D^{\gamma} u\left(s\left(x_{i}^{-\mu,-\mu}, \tilde{\theta}_{k}\right)\right) \omega_{k}^{-\mu, 0},
$$

where $\left\{\tilde{\theta}_{k}\right\}_{k=0}^{N}$ is the set of Jacobi-Gauss points corresponding to the weights $\left\{\omega_{k}^{-\mu, 0}\right\}_{k=0}^{N}$

We use

$$
u_{i} \approx u\left(x_{i}^{-\mu,-\mu}\right), \quad u_{i}^{\gamma} \approx D^{\gamma} u\left(x_{i}^{-\mu,-\mu}\right), \quad 0 \leq i \leq N
$$

and

$$
U(x)=\sum_{j=0}^{N} u_{j} F_{j}(x), \quad U^{\gamma}(x)=\sum_{j=0}^{N} u_{j}^{\gamma} F_{j}(x),
$$

where $F_{j}, j=0,1, \ldots, N$ is the Lagrange interpolation basis functions associated with $\left\{x_{i}^{-\mu,-\mu}\right\}_{i=0}^{N}$ which is the set of $N+1$ Jacobi-Gauss points. Combining the above equation and (11a) yields

$$
\begin{aligned}
u_{i}^{\gamma}= & u_{i}+\frac{1+x_{i}^{-\mu,-\mu}}{2} \sum_{k=0}^{N} K_{1}\left(x_{i}^{-\mu,-\mu}, s\left(x_{i}^{-\mu,-\mu}, \theta_{k}\right)\right) \sum_{j=0}^{N} u_{j} F_{j}\left(s\left(x_{i}^{-\mu,-\mu}, \theta_{k}\right)\right) \omega_{k}^{0,0} \\
& +\sum_{k=0}^{N} K_{2}\left(x_{i}^{-\mu,-\mu}, \theta_{k}\right) \sum_{j=0}^{N} u_{j} F_{j}\left(\theta_{k}\right) \omega_{k}^{0,0}+g\left(x_{i}^{-\mu,-\mu}\right)
\end{aligned}
$$




$$
\begin{aligned}
= & u_{i}+\frac{1+x_{i}^{-\mu,-\mu}}{2} \sum_{j=0}^{N} u_{j}\left(\sum_{k=0}^{N} K_{1}\left(x_{i}^{-\mu,-\mu}, s\left(x_{i}^{-\mu,-\mu}, \theta_{k}\right)\right) F_{j}\left(s\left(x_{i}^{-\mu,-\mu}, \theta_{k}\right)\right) \omega_{k}^{0,0}\right) \\
& +\sum_{j=0}^{N} u_{j} \sum_{k=0}^{N} K_{2}\left(x_{i}^{-\mu,-\mu}, \theta_{k}\right) F_{j}\left(\theta_{k}\right) \omega_{k}^{0,0}+g\left(x_{i}^{-\mu,-\mu}\right),
\end{aligned}
$$

$$
\begin{aligned}
u_{i} & =\frac{1}{\Gamma(\gamma)}\left(\frac{T\left(1+x_{i}^{-\mu,-\mu}\right)}{4}\right)^{\gamma}\left(\sum_{k=0}^{N} \sum_{j=0}^{N} u_{j}^{\gamma} F_{j}\left(s\left(x_{i}^{-\mu,-\mu}, \tilde{\theta}_{k}\right)\right) \omega_{k}^{-\mu, 0}\right)+u(-1) \\
& =\frac{1}{\Gamma(\gamma)}\left(\frac{T\left(1+x_{i}^{-\mu,-\mu}\right)}{4}\right)^{\gamma} \sum_{j=0}^{N} u_{j}^{\gamma}\left(\sum_{k=0}^{N} F_{j}\left(s\left(x_{i}^{-\mu,-\mu}, \tilde{\theta}_{k}\right)\right) \omega_{k}^{-\mu, 0}\right)+u(-1) .
\end{aligned}
$$

We can get the values of $\left\{u_{i}\right\}_{i=0}^{N}$ and $\left\{u_{i}^{\gamma}\right\}_{i=0}^{N}$ by solving the system of linear equations (16) and obtain the expressions of $U(x)$ and $U^{\gamma}(x)$ accordingly.

\section{Some useful lemmas}

In this section, we will provide some elementary lemmas, which are important for the derivation of the main results in the subsequent section.

Lemma 3.1 (see [9]). Assume that an $(N+1)$-point Gauss quadrature formula relative to the Jacobi weight is used to integrate the product $u \varphi$, where $u \in$ $H^{m}(I)$ with $I$ for some $m \geq 1$ and $\varphi \in \mathcal{P}_{N}$. Then there exists a constant $C$ independent of $N$ such that

$$
\left|\int_{-1}^{1} u(x) \varphi(x) d x-(u, \varphi)_{N}\right| \leq C N^{-m}|u|_{H_{\omega \alpha, \beta}^{m, N}(I)}\|\varphi\|_{L_{\omega \alpha, \beta}^{2}(I)},
$$

where

$$
\begin{aligned}
& |u|_{H_{\omega^{\alpha, \beta}}^{m, N}(I)}=\left(\sum_{j=\min (m, N+1)}^{m}\left\|u^{(j)}\right\|_{L_{\omega^{\alpha}, \beta}^{2}(I)}^{2}\right)^{1 / 2}, \\
& (u, \varphi)_{N}=\sum_{j=0}^{N} u\left(x_{j}\right) \varphi\left(x_{j}\right) \omega_{j} .
\end{aligned}
$$

Lemma 3.2 (see $[9,41]$ ). Assume that $u \in H_{\omega^{\alpha, \beta}}^{m, N}(I)$ and denote by $I_{N}^{\alpha, \beta} u$ its interpolation polynomial associated with the $(N+1)$ Jacobi-Gauss points $\left\{x_{j}\right\}_{j=0}^{N}$, namely,

$$
I_{N}^{\alpha, \beta} u=\sum_{i=0}^{N} u\left(x_{i}\right) F\left(x_{i}\right)
$$


Then the following estimates hold:

(19a)

$$
\left\|u-I_{N}^{\alpha, \beta} u\right\|_{L_{\omega^{\alpha, \beta}}^{2}(I)} \leq C N^{-m}|u|_{H_{\omega^{\alpha, \beta}}^{m, N}(I)},
$$

$$
\left\|u-I_{N}^{\alpha, \beta} u\right\|_{L^{\infty}(I)} \leq \begin{cases}C N^{\frac{1}{2}-m} \log N|u|_{H_{\omega^{c}}^{m, N}(I)}, & -1 \leq \alpha, \beta \leq-\frac{1}{2}, \\ C N^{1-\gamma-m}|u|_{H_{\omega^{c}}^{m, N}(I)}, & \gamma=\max (\alpha, \beta), \text { otherwise, }\end{cases}
$$

where $\omega^{c}=\omega^{-\frac{1}{2},-\frac{1}{2}}$ denotes the Chebyshev weight function.

Lemma 3.3 (see $[26]$ ). Assume that $\left\{F_{j}(x)\right\}_{j=0}^{N}$ are the $N$-th degree Lagrange basis polynomials associated with the Gauss points of the Jacobi polynomials. Then,

$$
\begin{aligned}
\left\|I_{N}^{\alpha, \beta}\right\|_{L^{\infty}(I)} & \leq \max _{x \in[-1,1]} \sum_{j=0}^{N}\left|F_{j}(x)\right| \\
& = \begin{cases}\mathcal{O}(\log N), & -1<\alpha, \beta \leq-\frac{1}{2}, \\
\mathcal{O}\left(N^{\gamma+\frac{1}{2}}\right), & \gamma=\max (\alpha, \beta), \text { otherwise. }\end{cases}
\end{aligned}
$$

Lemma 3.4 (Gronwall inequality, see [17] Lemma 7.1.1). Suppose $L \geq 0$, $0<\mu<1$, and $u$ and $v$ are a non-negative, locally integrable functions defined on $[-1,1]$ satisfying

$$
u(x) \leq v(x)+L \int_{-1}^{x}(x-\tau)^{-\mu} u(\tau) d \tau .
$$

Then there exists a constant $C=C(\mu)$ such that

$$
u(x) \leq v(x)+C L \int_{-1}^{x}(x-\tau)^{-\mu} v(\tau) d \tau \text { for } \quad-1 \leq x<1 .
$$

If a nonnegative integrable function $E(x)$ satisfies

$$
E(x) \leq L \int_{-1}^{x} E(s) d s+J(x), \quad-1<x \leq 1,
$$

where $J(x)$ is an integrable function, then

$$
\begin{aligned}
& \|E\|_{L^{\infty}(-1,1)} \leq C\|J\|_{L^{\infty}(-1,1)}, \\
& \|E\|_{L_{\omega-\alpha, \beta}^{p}(-1,1)} \leq C\|J\|_{L_{\omega-\alpha, \beta}^{p}(-1,1)}, \quad q \geq 1 .
\end{aligned}
$$

Lemma 3.5 (see $[30,31])$. For a nonnegative integer $r$ and $\kappa \in(0,1)$, there exists a constant $C_{r, \kappa}>0$ such that for any function $v \in C^{r, \kappa}([-1,1])$, there exists a polynomial function $\mathcal{T}_{N} v \in \mathcal{P}_{N}$ such that

$$
\left\|v-\mathcal{T}_{N} v\right\|_{L^{\infty}(I)} \leq C_{r, \kappa} N^{-(r+\kappa)}\|v\|_{r, \kappa}
$$

where $\|\cdot\|_{r, \kappa}$ is the standard norm in $C^{r, \kappa}([-1,1]), \mathcal{T}_{N}$ is a linear operator from $C^{r, \kappa}([-1,1])$ into $\mathcal{P}_{N}$, as stated in $[30,31]$. 
Lemma 3.6 (see [11]). Let $\kappa \in(0,1)$ and let $\mathcal{M}$ be defined by

$$
(\mathcal{M} v)(x)=\int_{-1}^{x}(x-\tau)^{-\mu} K(x, \tau) v(\tau) d \tau \text {. }
$$

Then, for any function $v \in C([-1,1])$, there exists a positive constant $C$ such that

$$
\frac{\left|\mathcal{M} v\left(x^{\prime}\right)-\mathcal{M} v\left(x^{\prime \prime}\right)\right|}{\left|x^{\prime}-x^{\prime \prime}\right|} \leq C \max _{x \in[-1,1]}|v(x)|,
$$

under the assumption that $0<\kappa<1-\mu$, for any $x^{\prime}, x^{\prime \prime} \in[-1,1]$ and $x^{\prime} \neq x^{\prime \prime}$. This implies that

$$
\|\mathcal{M} v\|_{0, \kappa} \leq C \max _{x \in[-1,1]}|v(x)|, \quad 0<\kappa<1-\mu .
$$

Lemma 3.7 (see [27]). For every bounded function $v$, there exists a constant $C$, independent of $v$, such that

$$
\sup _{N}\left\|\sum_{j=0}^{N} v\left(x_{j}\right) F_{j}(x)\right\|_{L_{\omega \alpha, \beta}^{2}(I)} \leq C \max _{x \in[-1,1]}|v(x)|,
$$

where $F_{j}(x), j=0,1, \ldots, N$, are the Lagrange interpolation basis functions associated with the Jacobi collocation points $\left\{x_{j}\right\}_{j=0}^{N}$.

Lemma 3.8 (see [22]). For all measurable functions $f \geq 0$, the following generalized Hardy's inequality

$$
\left(\int_{a}^{b}|(T f)(x)|^{q} u(x) d x\right)^{1 / q} \leq\left(\int_{a}^{b}|f(x)|^{p} v(x) d x\right)^{1 / p}
$$

holds if and only if

$$
\sup _{a<x<b}\left(\int_{x}^{b} u(t) d t\right)^{1 / q}\left(\int_{a}^{x} v^{1-p^{\prime}}(t) d t\right)^{1 / p^{\prime}}<\infty, \quad p^{\prime}=\frac{p}{p-1}
$$

for the case $1<p \leq q<\infty$. Here, $T$ is an operator of the form

$$
(T F)(x)=\int_{a}^{x} k(x, t) f(t) d t
$$

with $k(x, t)$ a given kernel, $u, v$ are nonnegative weight functions, and $-\infty \leq$ $a<b \leq \infty$.

\section{Convergence analysis}

This section is devoted to provide a convergence analysis for the numerical scheme. The goal is to show that the rate of convergence is exponential, i.e., that spectral accuracy can be obtained for the proposed approximations. Firstly, we will carry out our convergence analysis in the function space $L^{\infty}(I)$. 
Theorem 4.1. Let $u(x)$ be the exact solution of the fractional integro-differential equation (8), which is assumed to be sufficiently smooth. Assume that $U(x)$ and $U^{\gamma}(x)$ are obtained by using the spectral collocation scheme (16) together with a polynomial interpolation (15). If $\gamma$ associated with fractional order $0<$ $\gamma<1$ and $\mu=1-\gamma, u \in H_{\omega^{-\mu,-\mu}}^{m+1}(I)$, then

$$
\begin{aligned}
& \left\|U^{\gamma}-D^{\gamma} u\right\|_{L^{\infty}(I)} \\
\leq & \begin{cases}C N^{\gamma-\frac{1}{2}-m}\left(K^{*}\|u\|_{L^{2}(I)}+N^{\frac{1}{2}} \mathcal{U}\right), & \frac{1}{2}<\gamma<1, \\
C N^{-m} \log N\left(K^{*}\|u\|_{L^{2}(I)}+N^{\frac{1}{2}} \mathcal{U}\right), & 0<\gamma \leq \frac{1}{2},\end{cases} \\
\leq & \| \begin{array}{ll}
C \|^{\gamma-\frac{1}{2}-m}\left(K^{*}\|u\|_{L^{2}(I)}+N^{\frac{1}{2}} \mathcal{U}\right), & \frac{1}{2}<\gamma<1, \\
C N^{-m} \log N\left(K^{*}\|u\|_{L^{2}(I)}+N^{\frac{1}{2}} \mathcal{U}\right), & 0<\gamma \leq \frac{1}{2},
\end{array}
\end{aligned}
$$

provided that $N$ is sufficiently large, where $C$ is a constant independent of $N$ but depends on the bound of the function $K(x, s)$ and the index $\mu$,

$$
\begin{gathered}
K^{*}=\max _{x \in[-1,1]}\left|K_{1}(x, s(x, \theta))\right|_{H_{\omega^{0}, 0}^{m, N}(I)}+\max _{x \in[-1,1]}\left|K_{2}(x, \xi)\right|_{H_{\omega^{0}, 0}^{m, N}(I)}, \\
\mathcal{U}=\left|D^{\gamma} u\right|_{H_{\omega c}^{m, N}(I)}+|u|_{H_{\omega^{c}}^{m, N}(I)} .
\end{gathered}
$$

Proof. Since $U^{\gamma}(x)=\sum_{j=0}^{N} u_{j}^{\gamma} F_{j}(x) \in \mathcal{P}_{N}$, we have

$$
\int_{-1}^{1}(1-\theta)^{-\mu} U^{\gamma}(s) d s=\sum_{k=0}^{N} U^{\gamma}\left(s\left(x_{i}^{-\mu,-\mu}, \tilde{\theta}_{k}\right)\right) \omega_{k}^{-\mu, 0},
$$

the numerical scheme (16) can be written as

$$
\begin{aligned}
u_{i}^{\gamma}= & u_{i}+\frac{1+x_{i}^{-\mu,-\mu}}{2} \int_{-1}^{1} K_{1}\left(x_{i}^{-\mu,-\mu}, s\left(x_{i}^{-\mu,-\mu}, \theta\right)\right) U\left(s\left(x_{i}^{-\mu,-\mu}, \theta\right)\right) d \theta \\
& +\int_{-1}^{1} K_{2}\left(x_{i}^{-\mu,-\mu}, \xi\right) U(\xi) d \xi+I_{i, 1}+I_{i, 2}+g\left(x_{i}^{-\mu,-\mu}\right) \\
u_{i}= & \frac{1}{\Gamma(\gamma)}\left(\frac{T\left(1+x_{i}^{-\mu,-\mu}\right)}{4}\right)^{\gamma} \int_{-1}^{1}(1-\theta)^{-\mu} U^{\gamma}(s) d s+u(-1)
\end{aligned}
$$

where

$$
\begin{aligned}
I_{i, 1}= & \frac{1+x_{i}^{-\mu,-\mu}}{2} \sum_{k=0}^{N} K_{1}\left(x_{i}^{-\mu,-\mu}, s\left(x_{i}^{-\mu,-\mu}, \theta_{k}\right)\right) U\left(s\left(x_{i}^{-\mu,-\mu}, \theta_{k}\right)\right) \omega_{k}^{0,0} \\
& -\frac{1+x_{i}^{-\mu,-\mu}}{2} \int_{-1}^{1} K_{1}\left(x_{i}^{-\mu,-\mu}, s\left(x_{i}^{-\mu,-\mu}, \theta\right)\right) U\left(s\left(x_{i}^{-\mu,-\mu}, \theta\right)\right) d \theta \\
I_{i, 2}= & \sum_{k=0}^{N} K_{2}\left(x_{i}^{-\mu,-\mu}, \theta_{k}\right) U\left(\theta_{k}\right) \omega_{k}^{0,0}-\int_{-1}^{1} K_{2}\left(x_{i}^{-\mu,-\mu}, \xi\right) U(\xi) d \xi
\end{aligned}
$$


Let $e$ and $e^{\gamma}$ denote the error functions,

$$
e(x)=U(x)-u(x), \quad e^{\gamma}(x)=U^{\gamma}(x)-D^{\gamma} u(x) .
$$

Using the integration error estimates for the Jacobi-Gauss quadrature stated in Lemma 3.1, we have

(28)

$$
\begin{aligned}
& \left|I_{i, 1}(x)\right| \\
\leq & C N^{-m} \max _{x \in[-1,1]}\left|K_{1}(x, s(x, \theta))\right|_{H_{\omega^{0,0}}^{m, N}(I)}\|U\|_{L^{2}(I)} \\
\leq & C N^{-m} \max _{x \in[-1,1]}\left|K_{1}(x, s(x, \theta))\right|_{H_{\omega^{0}, 0}^{m, N}(I)}\left(\|u\|_{L^{2}(I)}+\|e\|_{L^{\infty}(I)}\right), \\
& \left|I_{i, 2}(x)\right| \\
\leq & C N^{-m} \max _{x \in[-1,1]}\left|K_{2}(x, \xi)\right|_{H_{\omega^{0}, 0}^{m, N}(I)}\|U\|_{L^{2}(I)} \\
\leq & C N^{-m} \max _{x \in[-1,1]}\left|K_{2}(x, \xi)\right|_{H_{\omega^{0}, 0}^{m, N}(I)}\left(\|u\|_{L^{2}(I)}+\|e\|_{L^{\infty}(I)}\right) .
\end{aligned}
$$

It follows from (9) and (11a) that

(30a) $u_{i}^{\gamma}=u_{i}+\int_{-1}^{x_{i}^{-\mu,-\mu}} K_{1}\left(x_{i}^{-\mu,-\mu}, s\right) U(s) d s+\int_{-1}^{1} K_{2}\left(x_{i}^{-\mu,-\mu}, \xi\right) U(\xi) d \xi$

$$
+I_{i, 1}+I_{i, 2}+g\left(x_{i}^{-\mu,-\mu}\right),
$$

(30b) $u_{i}=\frac{1}{\Gamma(\gamma)}\left(\frac{T\left(1+x_{i}^{-\mu,-\mu}\right)}{4}\right)^{\gamma} \int_{-1}^{x_{i}^{-\mu,-\mu}}\left(x_{i}^{-\mu,-\mu}-s\right)^{-\mu} U^{\gamma}(s) d s+u_{-1}$.

Multiplying by $F_{i}(x)$ both sides of (30) and summing from 0 to $N$ yield

$$
\begin{aligned}
U^{\gamma}(x)= & U(x)+I_{N}^{-\mu,-\mu} \int_{-1}^{x} K_{1}(x, s) u(s) d s+I_{N}^{-\mu,-\mu} \int_{-1}^{x} K_{1}\left(x^{-\mu,-\mu}, s\right) e(s) d s \\
& +I_{N}^{-\mu,-\mu} \int_{-1}^{1} K_{2}(x, s) u(s) d s+I_{N}^{-\mu,-\mu} \int_{-1}^{1} K_{2}\left(x^{-\mu,-\mu}, s\right) e(s) d s \\
& +I_{N}^{-\mu,-\mu}(g)+J_{1}(x)+J_{2}(x), \\
U(x)= & I_{N}^{-\mu,-\mu}\left(\frac{1}{\Gamma(\gamma)}\left(\frac{T\left(1+x_{i}^{-\mu,-\mu}\right)}{4}\right)^{\gamma} \int_{-1}^{x}(x-s)^{-\mu} D^{\gamma} u(s) d s\right) \\
& +I_{N}^{-\mu,-\mu}\left(\frac{1}{\Gamma(\gamma)}\left(\frac{T\left(1+x_{i}^{-\mu,-\mu}\right)}{4}\right)^{\gamma} \int_{-1}^{x}(x-s)^{-\mu} e^{\gamma}(s) d s\right)+u(-1),
\end{aligned}
$$

where

$$
J_{1}(x)=\sum_{i=0}^{N} I_{i, 1} F_{i}(x), \quad J_{2}(x)=\sum_{i=0}^{N} I_{i, 2} F_{i}(x) .
$$

It follows from (8) that

(32) $U^{\gamma}(x)=U(x)+I_{N}^{-\mu,-\mu}\left(D^{\gamma} u-u-g(x)\right)$ 


$$
\begin{aligned}
& +I_{N}^{-\mu,-\mu}\left(\int_{-1}^{x} K_{1}(x, s) e(s) d s+\int_{-1}^{1} K_{2}(x, s) e(s) d s\right) \\
& +I_{N}^{-\mu,-\mu}(g)+J_{1}(x)+J_{2}(x), \\
U(x)= & I_{N}^{-\mu,-\mu} u(x) \\
& +I_{N}^{-\mu,-\mu}\left(\frac{1}{\Gamma(\gamma)}\left(\frac{T\left(1+x_{i}^{-\mu,-\mu}\right)}{4}\right)^{\gamma} \int_{-1}^{x}(x-s)^{-\mu} e^{\gamma}(s) d s\right) .
\end{aligned}
$$

Then we have

(33a)

$$
e^{\gamma}(x)=e(x)+\int_{-1}^{x} K(x, s) e(s) d s+J_{1}(x)+J_{2}(x)+J_{3}(x)+J_{4}(x)+J_{5}(x),
$$

$$
e(x)=\frac{1}{\Gamma(\gamma)}\left(\frac{T\left(1+x_{i}^{-\mu,-\mu}\right)}{4}\right)^{\gamma} \int_{-1}^{x}(x-s)^{-\mu} e^{\gamma}(s) d s+J_{4}(x)+J_{6}(x),
$$

where

$$
\begin{aligned}
& J_{3}(x)=I_{N}^{-\mu,-\mu} D^{\gamma} u(x)-D^{\gamma} u(x), \\
& J_{4}(x)=I_{N}^{-\mu,-\mu} u(x)-u(x), \\
& J_{5}(x)=I_{N}^{-\mu,-\mu}\left(\int_{-1}^{x} K(x, s) e(s) d s\right)-\int_{-1}^{x} K(x, s) e(s) d s, \\
& J_{6}(x)=\frac{1}{\Gamma(\gamma)}\left(\frac{T\left(1+x_{i}^{-\mu,-\mu}\right)}{4}\right)^{\gamma}\left(I_{N}^{-\mu,-\mu} \int_{-1}^{x}(x-s)^{-\mu} e^{\gamma}(s) d s-\int_{-1}^{x}(x-s)^{-\mu} e^{\gamma}(s) d s\right) .
\end{aligned}
$$

Due to (33b) and using the Dirichlet's formula which states that

$$
\int_{-1}^{x} \int_{-1}^{\tau} \Phi(\tau, s) d s d \tau=\int_{-1}^{x} \int_{s}^{x} \Phi(\tau, s) d \tau d s
$$

provided the integral exists, we obtain

$$
\begin{aligned}
e^{\gamma}(x)= & e(x)+\frac{1}{\Gamma(\gamma)}\left(\frac{T\left(1+x_{i}^{-\mu,-\mu}\right)}{4}\right)^{\gamma} \int_{-1}^{x}\left(\int_{\tau}^{s} K(x, s) d s\right)(x-\tau)^{-\mu} e^{\gamma}(\tau) d \tau \\
& +\int_{-1}^{x} K(x, s)\left(J_{4}(s)+J_{6}(s)\right) d s+J_{1}(x)+J_{2}(x)+J_{3}(x)+J_{4}(x)+J_{5}(x),
\end{aligned}
$$

Denote $D_{1}:=\{(x, s):-1 \leq s \leq x, x \in[-1,1]\}$. We have

$$
\left|\int_{\tau}^{s} \frac{1}{\Gamma(\gamma)} K(x, s) d s\right| \leq \frac{2}{\Gamma(\gamma)} \max _{(x, s) \in D_{1}}|K(x, s)| \triangleq M,
$$

and then (34) gives

$$
\left|e^{\gamma}(x)\right| \leq M \int_{-1}^{x}(x-\tau)^{-\mu}\left|e^{\gamma}(\tau)\right| d \tau+|e(x)|
$$




$$
\begin{aligned}
& +\int_{-1}^{x} K(x, s)\left(\left|J_{4}(s)\right|+\left|J_{6}(s)\right|\right) d s \\
& +\left|J_{1}(x)\right|+\left|J_{2}(x)\right|+\left|J_{3}(x)\right|+\left|J_{4}(x)\right|+\left|J_{5}(x)\right| .
\end{aligned}
$$

It follows from the Gronwall inequality Lemma 3.4 that

$$
\left\|e^{\gamma}(x)\right\|_{L^{\infty}(I)} \leq C\left(\|e(x)\|_{L^{\infty}(I)}+\sum_{i=1}^{6}\left\|J_{i}\right\|_{L^{\infty}(I)}\right) .
$$

It follows from (33b) that

$$
\|e(x)\|_{L^{\infty}(I)} \leq C\left(\left\|e^{\gamma}(x)\right\|_{L^{\infty}(I)}+\sum_{i=4,6}\left\|J_{i}(x)\right\|_{L^{\infty}(I)}\right) .
$$

Then

$$
\begin{aligned}
& \left\|e^{\gamma}(x)\right\|_{L^{\infty}(I)} \leq C \sum_{i=1}^{6}\left\|J_{i}\right\|_{L^{\infty}(I)}, \\
& \|e(x)\|_{L^{\infty}(I)} \leq C \sum_{i=1}^{6}\left\|J_{i}\right\|_{L^{\infty}(I)} .
\end{aligned}
$$

Using Lemma 3.3, the estimates (28), and (37), we have

(40) $\left\|J_{1}\right\|_{L^{\infty}(I)}$

$\leq\left\{\begin{array}{l}C N^{\frac{1}{2}-\mu} \max _{0 \leq i \leq N}\left|I_{i, 1}\right|, \quad 0<\mu<\frac{1}{2}, \\ C \log N \max _{0 \leq i \leq N}\left|I_{i, 1}\right|, \frac{1}{2} \leq \mu<1,\end{array}\right.$

$\leq\left\{\begin{array}{l}C N^{\frac{1}{2}-\mu-m} \max _{x \in[-1,1]}\left|K_{1}(x, s(x, \theta))\right|_{H_{\omega^{0,0}}^{m, N}} \\ \left(\|u\|_{L^{2}(I)}+\left\|e^{\gamma}(x)\right\|_{L^{\infty}(I)}+\sum_{i=4,6}\left\|J_{i}(x)\right\|_{L^{\infty}(I)}\right), 0<\mu<\frac{1}{2}, \\ C N^{-m} \log N \max _{x \in[-1,1]}\left|K_{1}(x, s(x, \theta))\right|_{H_{\omega^{0,0}}^{m, N}} \\ \left(\|u\|_{L^{2}(I)}+\left\|e^{\gamma}(x)\right\|_{L^{\infty}(I)}+\sum_{i=4,6}\left\|J_{i}(x)\right\|_{L^{\infty}(I)}\right), \frac{1}{2} \leq \mu<1 .\end{array}\right.$

(41) $\left\|J_{2}\right\|_{L^{\infty}(I)}$

$\leq\left\{\begin{array}{l}C N^{\frac{1}{2}-\mu} \max _{0 \leq i \leq N}\left|I_{i, 2}\right|, \quad 0<\mu<\frac{1}{2}, \\ C \log N \max _{0 \leq i \leq N}\left|I_{i, 2}\right|, \quad \frac{1}{2} \leq \mu<1,\end{array}\right.$

$\leq\left\{\begin{array}{l}C N^{\frac{1}{2}-\mu-m} \max _{x \in[-1,1]}\left|K_{2}(x, s(x, \theta))\right|_{H^{m, N}, 0} \\ \left(\|u\|_{L^{2}(I)}+\left\|e^{\gamma}(x)\right\|_{L^{\infty}(I)}+\sum_{i=4,6}\left\|J_{i}(x)\right\|_{L^{\infty}(I)}\right), 0<\mu<\frac{1}{2}, \\ C N^{-m} \log N \max _{x \in[-1,1]}\left|K_{2}(x, s(x, \theta))\right|_{H_{\omega^{0,0}}^{m, N}} \\ \left(\|u\|_{L^{2}(I)}+\left\|e^{\gamma}(x)\right\|_{L^{\infty}(I)}+\sum_{i=4,6}\left\|J_{i}(x)\right\|_{L^{\infty}(I)}\right), \frac{1}{2} \leq \mu<1 .\end{array}\right.$ 
Due to Lemma 3.2,

$$
\begin{aligned}
& \left\|J_{3}\right\|_{L^{\infty}(I)} \leq\left\{\begin{array}{l}
C N^{1-\mu-m}\left|D^{\gamma} u\right|_{H_{\omega^{c}}^{m, N}(I)}, 0<\mu<\frac{1}{2}, \\
C N^{\frac{1}{2}-m} \log N\left|D^{\gamma} u\right|_{H_{\omega^{c}}^{m, N}(I)}, \frac{1}{2} \leq \mu<1,
\end{array}\right. \\
& \left\|J_{4}\right\|_{L^{\infty}(I)} \leq\left\{\begin{array}{l}
C N^{1-\mu-m}|u|_{H_{\omega c}^{m, N}(I)}, 0<\mu<\frac{1}{2}, \\
C N^{\frac{1}{2}-m} \log N|u|_{H_{\omega c}^{m, N}(I)}, \quad \frac{1}{2} \leq \mu<1 .
\end{array}\right.
\end{aligned}
$$

By virtue of Lemma 3.2(19b) with $m=1$,

$$
\begin{aligned}
& \left\|J_{5}\right\|_{L^{\infty}(I)} \\
\leq & \left\{\begin{array}{l}
C N^{-\mu}\|e\|_{L^{\infty}(I)}, \quad 0<\mu<\frac{1}{2} \\
C N^{-\frac{1}{2}}\|e\|_{L^{\infty}(I)}, \frac{1}{2} \leq \mu<1,
\end{array}\right. \\
\leq & \left\{\begin{array}{l}
C N^{-\mu}\left(\left\|e^{\gamma}\right\|_{L^{\infty}(I)}+\left\|J_{3}\right\|_{L^{\infty}(I)}+\left\|J_{5}\right\|_{L^{\infty}(I)}\right), \quad 0<\mu<\frac{1}{2}, \\
C N^{-\frac{1}{2}}\left(\left\|e^{\gamma}\right\|_{L^{\infty}(I)}+\left\|J_{3}\right\|_{L^{\infty}(I)}+\left\|J_{5}\right\|_{L^{\infty}(I)}\right), \frac{1}{2} \leq \mu<1 .
\end{array}\right.
\end{aligned}
$$

We now estimate the term $J_{5}(x)$. It follows from Lemma 3.5 and Lemma 3.6 with $K(x, \tau)=\frac{1}{\Gamma(\gamma)}$ that

$$
\begin{aligned}
\left\|J_{6}\right\|_{L^{\infty}(I)} & =\left\|\left(I_{N}^{-\mu,-\mu}-I\right) \mathcal{M} e^{\gamma}\right\|_{L^{\infty}(I)} \\
& =\left\|\left(I_{N}^{-\mu,-\mu}-I\right)\left(\mathcal{M} e^{\gamma}-\mathcal{T}_{N} \mathcal{M} e^{\gamma}\right)\right\|_{L^{\infty}(I)} \\
& \leq\left(1+\left\|I_{N}^{-\mu,-\mu}\right\|_{L^{\infty}(I)}\right) C N^{-k}\left\|\mathcal{M} e^{\gamma}\right\|_{0, \kappa} \\
& \leq\left\{\begin{array}{l}
C N^{\frac{1}{2}-\mu-\kappa}\left\|e^{\gamma}\right\|_{L^{\infty}(I)}, 0<\mu<\frac{1}{2}, \\
C N^{-\kappa} \log N\left\|e^{\gamma}\right\|_{L^{\infty}(I)}, \frac{1}{2} \leq \mu<1,
\end{array}\right.
\end{aligned}
$$

where in the last step we have used Lemma 3.6 under the following assumption

$$
\begin{cases}\frac{1}{2}-\mu<\kappa<1-\mu, & \text { when } \quad 0<\mu<\frac{1}{2}, \\ 0<\kappa<1-\mu, & \text { when } \quad \frac{1}{2} \leq \mu<1,\end{cases}
$$

provided that $N$ is sufficiently large. Combining (40), (42), (43) and (44) gives

$$
\begin{aligned}
& \left\|U^{\gamma}(x)-u^{\gamma}(x)\right\|_{L^{\infty}(I)} \\
\leq & \left\{\begin{array}{l}
C N^{\frac{1}{2}-\mu-m}\left(K^{*}\|u\|_{L^{2}(I)}+N^{\frac{1}{2}}\left(\left|D^{\gamma} u\right|_{H_{\omega^{c}}^{m, N}(I)}+|u|_{H_{\omega^{c}}^{m, N}(I)}\right)\right), 0<\mu<\frac{1}{2}, \\
C N^{-m} \log N\left(K^{*}\|u\|_{L^{2}(I)}+N^{\frac{1}{2}}\left(\left|D^{\gamma} u\right|_{H_{\omega c}^{m, N}(I)}+|u|_{H_{\omega^{c}}^{m, N}(I)}\right)\right), \quad \frac{1}{2} \leq \mu<1 .
\end{array}\right. \\
& \|U(x)-u(x)\|_{L^{\infty}(I)} \\
\leq & \left\{\begin{array}{l}
C N^{\frac{1}{2}-\mu-m}\left(K^{*}\|u\|_{L^{2}(I)}+N^{\frac{1}{2}}\left(\left|D^{\gamma} u\right|_{H_{\omega^{c}}^{m, N}(I)}+|u|_{H_{\omega^{c}}^{m, N}(I)}\right)\right), 0<\mu<\frac{1}{2}, \\
C N^{-m} \log N\left(K^{*}\|u\|_{L^{2}(I)}+N^{\frac{1}{2}}\left(\left|D^{\gamma} u\right|_{H_{\omega^{c}}^{m, N}(I)}+|u|_{H_{\omega^{c}}^{m, N}(I)}\right)\right), \frac{1}{2} \leq \mu<1 .
\end{array}\right.
\end{aligned}
$$

Using $\gamma=1-\mu$, we have the desired estimate (23) and (24).

Next, we will derive the error estimates in the function space $L_{\omega^{-\mu, \mu}}^{2}(I)$. 
Theorem 4.2. If the hypotheses given in Theorem 4.1 hold, then

$$
\begin{aligned}
& \left\|U^{\gamma}(x)-u^{\gamma}(x)\right\|_{L_{\omega-\mu,-\mu}^{2}}(I) \\
\leq & \begin{cases}C N^{-m}\left(V_{1}+N^{\gamma-\frac{1}{2}-\kappa} V_{2}+N^{\gamma-\kappa} \mathcal{U}\right), & \frac{1}{2}<\gamma<1, \\
C N^{-m}\left(V_{1}+N^{-\kappa} \log N V_{2}+N^{\frac{1}{2}-\kappa} \log N \mathcal{U}\right), & 0<\gamma \leq \frac{1}{2},\end{cases}
\end{aligned}
$$

$$
\begin{aligned}
& \|U(x)-u(x)\|_{L_{\omega-\mu,-\mu}^{2}(I)} \\
\leq & \begin{cases}C N^{-m}\left(V_{1}+N^{\gamma-\frac{1}{2}-\kappa} V_{2}+N^{\gamma-\kappa} \mathcal{U}\right), & \frac{1}{2}<\gamma<1, \\
C N^{-m}\left(V_{1}+N^{-\kappa} \log N V_{2}+N^{\frac{1}{2}-\kappa} \log N \mathcal{U}\right), & 0<\gamma \leq \frac{1}{2},\end{cases}
\end{aligned}
$$

for any $\kappa \in(0, \gamma)$, provided that $N$ is sufficiently large and $C$ is a constant independent of $N$, where

$$
\begin{aligned}
V_{1} & =K^{*}\left(\|u\|_{L^{2}(I)}+\left|D^{\gamma} u\right|_{H_{\omega^{c}}^{1, N}(I)}+|u|_{H_{\omega^{c}}^{1, N}(I)}\right), \\
V_{2} & =K^{*}\|u\|_{L^{2}(I)}, \\
\mathcal{U} & =\left|D^{\gamma} u\right|_{H_{\omega^{c}}^{m, N}(I)}+|u|_{H_{\omega^{c}}^{m, N}(I)} .
\end{aligned}
$$

Proof. By using the generalization of the Gronwall inequality Lemma 3.4 and the Hardy inequality Lemma 3.8, it follows from (33) that

$$
\left\|e^{\gamma}\right\|_{L_{\omega}^{2}-\mu,-\mu}(I) \leq C \sum_{i=1}^{6}\left\|J_{i}\right\|_{L_{\omega}^{2}-\mu,-\mu}(I),
$$

and

$$
\|e\|_{L_{\omega-\mu,-\mu}^{2}(I)} \leq C \sum_{i=1}^{6}\left\|J_{i}\right\|_{L_{\omega^{-\mu,-\mu}}^{2}(I)} .
$$

Now, using Lemma 3.7, we have

$$
\begin{aligned}
& \left\|J_{1}\right\|_{L_{\omega-\mu,-\mu}^{2}(I)} \\
\leq & C \max _{x \in[-1,1]}\left|I_{i, 1}(x)\right| \\
\leq & C N^{-m} \max _{x \in[-1,1]}\left|K_{1}(x, s(x, \theta))\right|_{H_{\omega^{0}, 0}^{m, N}(I)}\left(\|u\|_{L^{2}(I)}+\|e\|_{L^{\infty}(I)}\right) . \\
& \left\|J_{2}\right\|_{L_{\omega}^{2}-\mu,-\mu}(I) \\
\leq & C \max _{x \in[-1,1]}\left|I_{i, 2}(x)\right| \\
\leq & C N^{-m} \max _{x \in[-1,1]}\left|K_{2}(x, \xi)\right|_{H_{\omega^{0}, 0}^{m, N}(I)}\left(\|u\|_{L^{2}(I)}+\|e\|_{L^{\infty}(I)}\right) .
\end{aligned}
$$

By the convergence result in Theorem $4.1(m=1)$, we have

$$
\|e\|_{L^{\infty}(I)} \leq C\left(\left|D^{\gamma} u\right|_{H_{\omega^{c}}^{1, N}(I)}+|u|_{H_{\omega^{c}}^{1, N}(I)}+\|u\|_{L^{2}(I)}\right) .
$$


So that

(51) $\sum_{i=1}^{2}\left\|J_{i}\right\|_{L_{\omega}^{2}-\mu,-\mu}(I) \leq C N^{-m} K^{*}\left(\left|D^{\gamma} u\right|_{H_{\omega c}^{1, N}(I)}+|u|_{H_{\omega c}^{1, N}(I)}+\|u\|_{L^{2}(I)}\right)$.

Due to Lemma 3.2(19a),

$$
\begin{aligned}
& \left\|J_{3}\right\|_{L_{\omega}^{3}-\mu,-\mu}(I) \leq C N^{-m}\left|D^{\gamma} u\right|_{H_{\omega c}^{m, N}(I)}, \\
& \left\|J_{4}\right\|_{L_{\omega}^{4}-\mu,-\mu}(I) \leq C N^{-m}|u|_{H_{\omega c}^{m, N}(I)} .
\end{aligned}
$$

By virtue of Lemma 3.2(19a) with $m=1$,

$$
\begin{aligned}
\left\|J_{5}\right\|_{L_{\omega-\mu,-\mu}^{2}(I)} & \leq C N^{-1}\left|\int_{-1}^{x} K(x, s) e(s) d s\right|_{H_{\omega-\mu,-\mu}^{1, N}(I)} \\
& \leq C N^{-1}\|e\|_{L_{\omega-\mu,-\mu}^{2}(I)} .
\end{aligned}
$$

Finally, it follows from Lemma 3.5 and Lemma 3.7 that

$$
\begin{aligned}
& \left\|J_{6}\right\|_{L_{\omega-\mu,-\mu}^{2}}(I)
\end{aligned}
$$

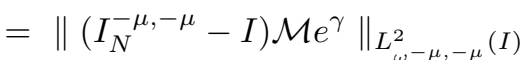

$$
\begin{aligned}
& =\|\left(I_{N}^{-\mu,-\mu}-I\right)\left(\mathcal{M} e^{\gamma}-\mathcal{T}_{N} e^{\gamma} \|_{L_{\omega}^{2}-\mu,-\mu}(I)\right. \\
& \leq\left\|I_{N}^{-\mu,-\mu}\left(\mathcal{M} e^{\gamma}-\mathcal{T}_{N} e^{\gamma}\right)\right\|_{L_{\omega}^{2}-\mu,-\mu}(I)+\left\|\mathcal{M} e^{\gamma}-\mathcal{T}_{N} e^{\gamma}\right\|_{L_{\omega}^{2}-\mu,-\mu}(I) \\
& \leq C\left\|\mathcal{M} e^{\gamma}-\mathcal{T}_{N} e^{\gamma}\right\|_{L^{\infty}(I)} \\
& \leq C N^{-\kappa}\left\|\mathcal{M} e^{\gamma}\right\|_{0, \kappa} \\
& \leq C N^{-\kappa}\left\|e^{\gamma}\right\|_{L^{\infty}(I)}
\end{aligned}
$$

where, in the last step we used Lemma 3.6 for any $\kappa \in(0,1-\mu)$. By the convergence result in Theorem 4.1, we obtain that

$$
\sum_{i=1}^{6}\left\|J_{i}\right\|_{L_{\omega}^{2}-\mu,-\mu}(I) \leq \begin{cases}C N^{\frac{1}{2}-\mu-m-\kappa}\left(K^{*}\|u\|_{L^{2}(I)}+N^{\frac{1}{2}} \mathcal{U}\right), & 0<\mu<\frac{1}{2} \\ C N^{-m-\kappa} \log N\left(K^{*}\|u\|_{L^{2}(I)}+N^{\frac{1}{2}} \mathcal{U}\right), & \frac{1}{2} \leq \mu<1\end{cases}
$$

for $N$ sufficiently large and for any $\kappa \in(0,1-\mu)$. The desired estimates (45) and (46) are obtained by combining (47), (48), (51), (52), (53), (55) and $\gamma=1-\mu$.

\section{Algorithm implementation and numerical results}

Writing $U_{N}=\left(u_{0}, u_{1}, \ldots, u_{N}\right)^{T}$ and $U_{N}^{\gamma}=\left(u_{0}^{\gamma}, u_{1}^{\gamma}, \ldots, u_{N}^{\gamma}\right)^{T}$, we obtain the following equations of the matrix form from (16):

$$
\begin{aligned}
& U_{N}^{\gamma}=(E+A+B) U_{N}+G_{N}, \\
& U_{N}=U_{-1}+C U_{N}^{\gamma},
\end{aligned}
$$


where

$E$ is the identity matrix,

$$
\begin{aligned}
A_{i j} & =\frac{1+x_{i}^{-\mu,-\mu}}{2} \sum_{k=0}^{N} K_{1}\left(x_{i}^{-\mu,-\mu}, s\left(x_{i}^{-\mu,-\mu}, \theta_{k}\right)\right) F_{j}\left(s\left(x_{i}^{-\mu,-\mu}, \theta_{k}\right)\right) \omega_{k}^{0,0}, \\
B_{i j} & =\sum_{k=0}^{N} K_{2}\left(x_{i}^{-\mu,-\mu}, \theta_{k}\right) F_{j}\left(\theta_{k}\right) \omega_{k}^{0,0} \\
C_{i j} & =\frac{1}{\Gamma(\gamma)}\left(\frac{T\left(1+x_{i}^{-\mu,-\mu}\right)}{4}\right)^{\gamma} \sum_{k=0}^{N} F_{j}\left(s\left(x_{i}^{-\mu,-\mu}, \tilde{\theta}_{k}\right)\right) \omega_{k}^{-\mu, 0}, \\
G_{N} & =\left(g\left(x_{0}^{-\mu,-\mu}\right), g\left(x_{1}^{-\mu,-\mu}\right), \ldots, g\left(x_{N}^{-\mu,-\mu}\right)\right)^{T}, \\
U_{-1} & =u(-1) \times(1,1, \ldots, 1)^{T} .
\end{aligned}
$$

Example 1. Consider the following fractional integro-differential equation

$$
\begin{aligned}
D^{0.75} y(t)= & \frac{6 t^{2.25}}{\Gamma(3.25)}-\frac{t}{4}+\frac{1}{5}-\frac{1}{5} t^{2} e^{t} y(t) \\
& +\int_{0}^{t} e^{t} \tau y(\tau) d \tau+\int_{0}^{1}(t-\varsigma) y(\varsigma) d \varsigma, \\
y(0)= & 0 .
\end{aligned}
$$

The corresponding exact solution is given by $y(t)=t^{3}, D^{0.75} y(t)=\frac{6 t^{2.25}}{\Gamma(3.25)}$.

Figure 1 presents the approximate and exact solutions on the left-hand side and presents the approximate and exact derivatives on the right-hand side, which are found in excellent agreement. In Figure 2, the numerical errors are plotted for $2 \leq N \leq 20$ in both $L^{\infty}$ and $L_{\omega^{-\mu,-\mu}}^{2}$ norms. As expected, an exponential rate of convergence is observed for the problem, which confirmed our theoretical predictions.

Example 2. Our last example is about a nonlinear problem in one-dimension. Consider the following fractional integro-differential equation,

$$
\begin{aligned}
& D^{0.5} y(t)=f(t) y(t)+g(t)+\frac{1}{2}+\sqrt{t} \int_{0}^{t} y^{2}(\tau) d \tau+\int_{0}^{1} y(\varsigma) d \varsigma, \\
& y(0)=0
\end{aligned}
$$

with

$$
f(t)=2 \sqrt{t}+2 t^{\frac{3}{2}}-\left(\sqrt{t}+t^{\frac{3}{2}}\right) \ln (1+t), \quad g(t)=\frac{2 \operatorname{arcsinh}(\sqrt{t})}{\sqrt{\pi(1+t)}}-2 t^{\frac{3}{2}} .
$$

The exact solution is $y(t)=\ln (1+t)$.

This is a nonlinear problem. The numerical scheme (16) leads to a nonlinear system for $\left\{u_{i}\right\}_{i=1}^{N}$, and a proper solver for the nonlinear system (e.g., Newton method) should be used. The numerical results can be seen from Figure 3. 

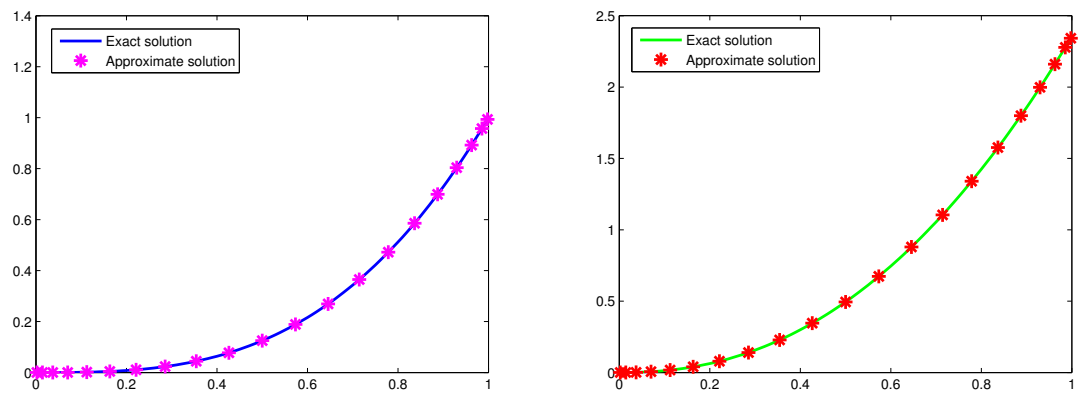

Figure 1. Example 1: Comparison between approximate solution and exact solution $y(t)$ (left), approximate fraction derivative and exact derivative $D^{0.75} y(t)$ (right)
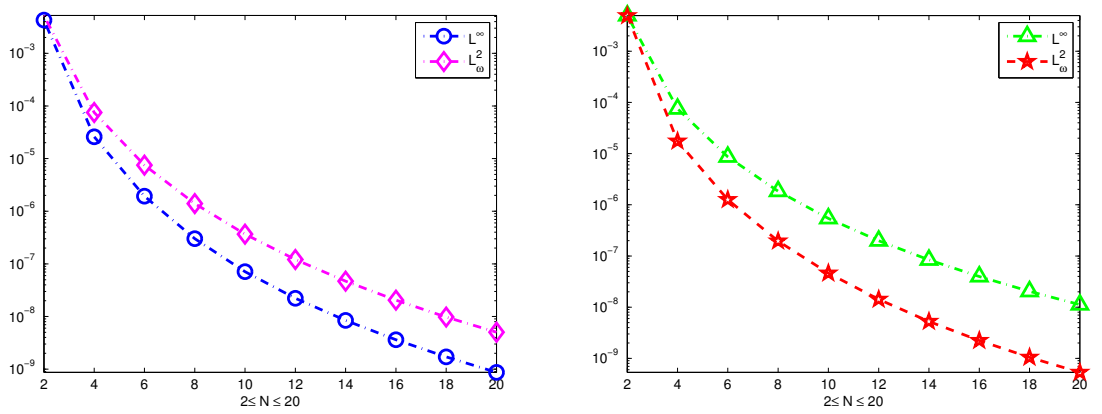

Figure 2. Example 1: The errors of numerical and exact solution $y(t)$ (left) and the errors of numerical and exact solution $D^{0.75} y(t)$ (right) versus the number of collocation points in $L^{\infty}$ and $L_{\omega}^{2}$ norms.

These results indicate that the spectral accuracy is obtained for this problem , although the given functions $f(t)$ and $g(t)$ are not very smooth.

Example 3. Consider the following fractional integro-differential equation

$$
\begin{aligned}
D^{\alpha} y(t)= & 2+2 t-2 y(t)+t(1+2 t) \int_{0}^{t} e^{\tau(t-\tau)} y(\tau) d \tau \\
& +\int_{0}^{1} e^{\varsigma(t-\varsigma)} y(\varsigma) d \varsigma, \\
y(0)=1 &
\end{aligned}
$$

when $\alpha=1$, the exact solution of (59) is $y(t)=e^{t^{2}}$. 

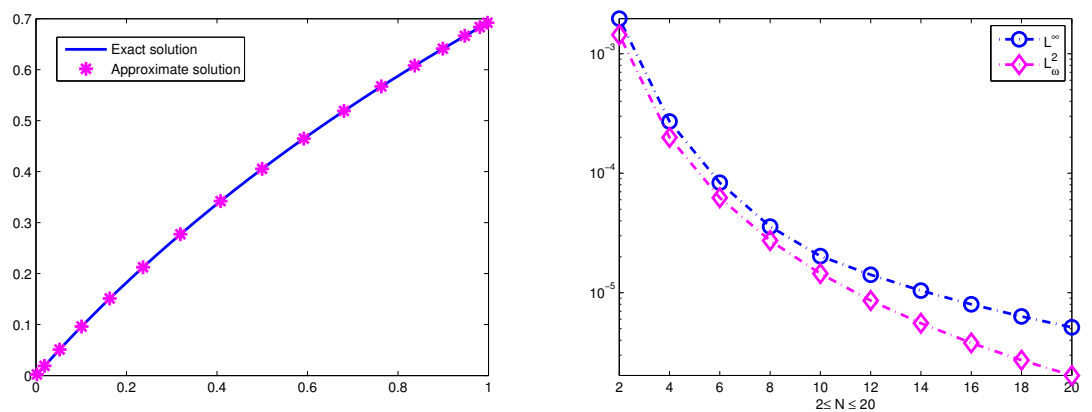

Figure 3. Example 2: Comparison between approximate solution and exact solution of $y(t)$ (left). The errors of numerical and exact solution $y(t)$ versus the number of collocation points in $L^{\infty}$ and $L_{\omega}^{2}$ norms (right).
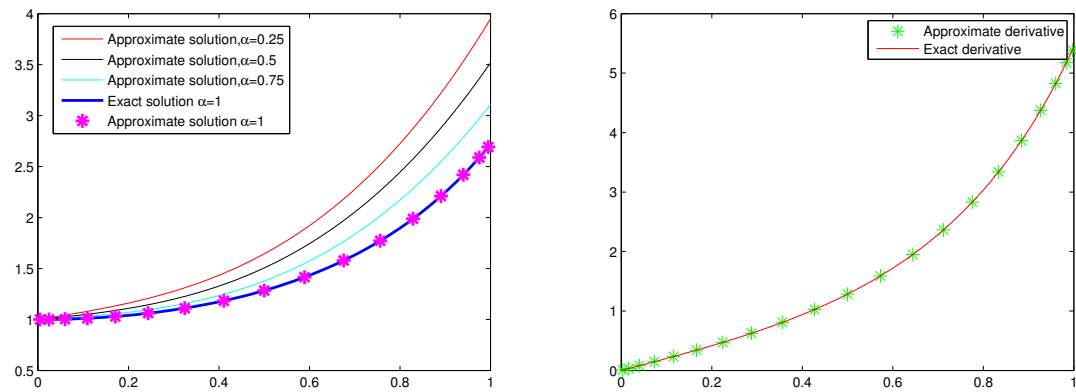

Figure 4. Example 3: Approximation solutions with different $\alpha$ and exact solution of $y(t)$ with $\alpha=1$ (left). Comparison between approximate solution and exact solution of $y^{\prime}(t)$.

In the only case of $\alpha=1$, we know the exact solution. We have reported the obtained numerical results for $N=20$ and $\alpha=0.25,0.5,0.75,1$ in Figure 4 . We can see that, as $\alpha$ approaches 1 , the numerical solutions converges to the analytical solution $y(t)=e^{t^{2}}$, i.e., in the limit, the solution of fractional integro differential equations approaches to that of the integer order integro differential equations. In Figure 5, we plot the resulting errors versus the number $N$ of the steps. This figure shows the exponential rate of convergence predicted by the proposed method. 

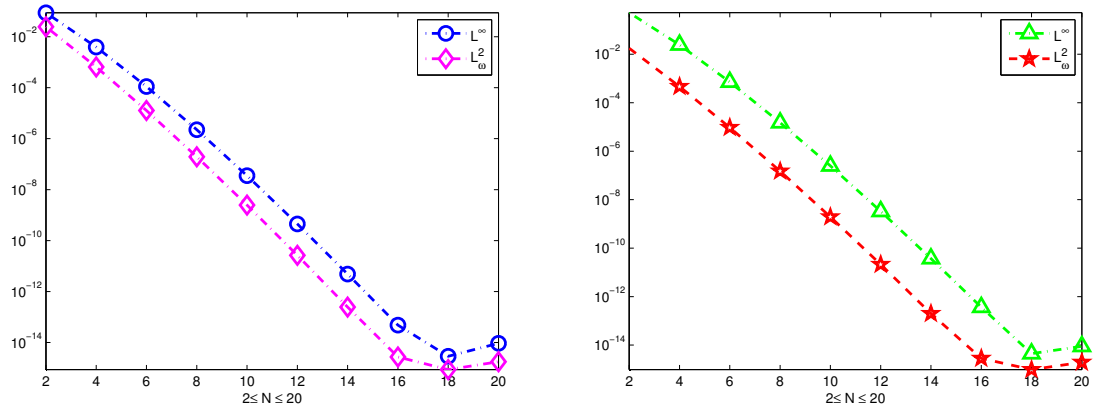

Figure 5. Example 3: The errors of numerical and exact solution $y(t)$ (left) and the errors of numerical and exact solution $y^{\prime}(t)$ (right) versus the number of collocation points in $L^{\infty}$ and $L_{\omega}^{2}$ norms.

\section{References}

[1] P. Agrawal and P. Kumar, Comparison of five numerical schemes for fractional differential equations, Advances in fractional calculus, 43-60, Springer, Dordrecht, 2007.

[2] W. M. Ahmad and R. EL-Khazali, Fractional-order dynamical models of love, Chaos Solitons Fractals 33 (2007), no. 4, 1367-1375.

[3] P. Baratella and A. Orsi, A new approach to the numerical solution of weakly singular Volterra integral equations, J. Comput. Appl. Math. 163 (2004), no. 2, 401-418.

[4] A. H. Bhrawy and M. A. Alghamdi, A shifted Jacobi-Gauss-Lobatto collocation method for solving nonlinear fractional Langevin equation involving two fractional orders in different intervals, Bound. Value Probl. 1 (2012), no. 62, 1-13.

[5] A. H. Bhrawy and A. S. Alofi, The operational matrix of fractional integration for shifted Chebyshev polynomials, Appl. Math. Lett. 26 (2013), no. 1, 25-31.

[6] A. H. Bhrawy and M. Alshomrani, A shifted Legendre spectral method for fractionalorder multi-point boundary value problems, Advan Differ Eqs. 2012 (2012), 1-8.

[7] A. H. Bhrawy, A. S. Alofi, and S. S. Ezz-Eldien, A quadrature tau method for fractional differential equations with variable coefficients, Appl. Math. Lett. 24 (2011), no. 12, 2146-2152.

[8] A. H. Bhrawy, M. M. Tharwat, and A. Yildirim, A new formula for fractional integrals of Chebyshev polynomials: Application for solving multi-term fractional differential equations, Appl. Math. Model. 37 (2013), no. 6, 4245-4252.

[9] C. Canuto, M. Y. Hussaini, and A. Quarteroni, Spectral Methods, Fundamentals in single domains, Springer-Verlag, Berlin, 2006.

[10] Y. Chen and T. Tang, Convergence analysis of the Jacobi spectral-collocation methods for Volterra integral equation with a weakly singular kernel, Math. Comp. 79 (2010), no. $269,147-167$.

[11] D. Colton and R. Kress, Inverse Acoustic and Electromagnetic Scattering Theory, Springer-Verlag, Heidelberg, 2nd Edition, 1998

[12] E. H. Doha, A. H. Bhrawy, and S. S. Ezz-Eldien, Efficient Chebyshev spectral methods for solving multi-term fractional orders differential equations, Appl. Math. Model. 35 (2011), no. $12,5662-5672$. 
[13] _ A Chebyshev spectral method based on operational matrix for initial and boundary value problems of fractional order, Comput. Math. Appl. 62 (2011), no. 5, 23642373.

[14] _ A new Jacobi operational matrix: an application for solving fractional differential equations, Appl. Math. Model. 36 (2012), no. 10, 4931-4943.

[15] J. H. He, Nonlinear oscillation with fractional derivative and its applications, In: International Conference on Vibrating Engineering, Dalian, China, 1998, 288-291.

[16] _ Some applications of nonlinear fractional differential equations and therir approximations, Bull.Sci. Technol. 15 (1999), 86-90.

[17] D. Henry, Geometric Theory of Semilinear Parabolic Equations, Springer-Verlag, 1989.

[18] F. Huang and F. Liu, The time fractional diffusion equation and the advection-dispersion equation, ANZIAM J. 46 (2005), 317-330.

[19] H. Jafari and S. A. Yousefi, Application of Legendre wavelets for solving fractional differential equations, Comput. Math. Appl. 62 (2011), no. 3, 1038-1045.

[20] M. M. Khader and A. S. Hendy, An efficient numerical scheme for solving fractional optimal control problems, Int. J. Nonlinear Sci. 14 (2012), no. 3, 287-297.

[21] M. M. Khader, N. H. Sweilam, and A. M. S. Mahdy, An efficient numerical method for solving the fractional diffusion equation, J. Appl. Math. Bioinf. 1 (2011), no. 2, 1-12.

[22] A. Kufner and L. E. Persson, Weighted Inequalities of Hardy Type, World Scientific, New York, 2003

[23] Y. L. Li, Haar wavelet operational matrix of fractional order integration and its applications in solving the fractional order differential equations, Appl. Math. Comput. 216 (2010), no. 8, 2276-2285.

[24] Y. Luchko and R. Gorenflo, The initial value problem for some fractional differential equations with the Caputo derivatives, Preprint series A08-98, Fachbreich Mathematik und Informatik, Freic Universitat Berlin, 1998.

[25] F. Mainardi, Fractional calculus: some basic problems in continuum and statistical mechanics, Fractals and fractional calculus in continuum mechanics (Udine, 1996), 291-348, CISM Courses and Lectures, 378, Springer, Vienna, 1997.

[26] G. Mastroianni and D. Occorsto, Optimal systems of nodes for Lagrange interpolation on bounded intervals: a survey, J. Comput. Appl. Math. 134 (2001), no. 1-2, 325-341.

[27] P. Nevai, Mean convergence of Lagrange interpolation. III, Trans. Amer. Math. Soc. 282 (1984), no. 2, 669-698.

[28] A. Pedas and E. Tamme, Piecewise polynomial collocation for linear boundary value problems of fractional differential equations, J. Comput. Appl. Math. 236 (2012), no. 13, 3349-3359.

[29] I. Podlubny, Fractional Differential Equations, Academic Press, New York, 1999.

[30] D. L. Ragozin, Polynomial approximation on compact manifolds and homogeneous spaces, Trans. Amer. Math. Soc. 150 (1970), 41-53.

[31] _ Constructive polynomial approximation on spheres and projective spaces, Trans. Amer. Math. Soc. 162 (1971), 157-170.

[32] E. A. Rawashdeh, Legendre wavelets method for fractional integro-differential equations, Appl. Math. Sci. 5 (2011), no. 49-52, 2467-2474.

[33] - Numerical solution of fractional integro-differential equations by collocation method, Appl. Math. Comput. 176 (2006), no. 1, 1-6.

[34] M. Rehman and R. A. Khan, The Legendre wavelet method for solving fractional differential equations, Commun. Nonlinear Sci. Numer. Simul. 16 (2011), no. 11, 4163-4173.

[35] A. Saadatmandi and M. Dehghan, A new operational matrix for solving fractional-order differential equations, Comput. Math. Appl. 59 (2010), no. 3, 1326-1336.

[36] _ A Legendre collocation method for fractional integro-differential equations, J. Vib. Control 17 (2011), no. 13, 2050-2058. 
[37] G. Samko, A. A. Kilbas, and O. I. Marichev, Fractional integrals and derivatives: theory and applications, Gordon \& Breach, Yverdon, 1993.

[38] N. T. Shawagfeh, Analytical approximate solutions for nonlinear fractional differential equations, Appl. Math. Comput. 131 (2002), no. 2-3, 517-529.

[39] N. H. Sweilam and M. M. Khader, A Chebyshev pseudo-spectral method for solving fractional-order integro-differential equations, ANZIAM J. 51 (2010), no. 4, 464-475.

[40] N. H. Sweilam, M. M. Khader, and R. F. Al-Bar, Homotopy perturbation method for linear and nonlinear system of fractional integro-differential equations, Int. J. Comput. Math. Numer. Simul. 1 (2008), no. 1, 73-87.

[41] Y. Wei and Y. Chen, Convergence analysis of the spectral methods for weakly singular Volterra integro-differential equations with smooth solutions, Adv. Appl. Math. Mech. 4 (2012), no. 1, 1-20.

YIN YANG

Hunan Key Laboratory For COMPUtation AND Simulation in Science and Engineering

Xiangtan University

Xiangtan 411105, P. R. China

E-mail address: yangyinxtu@xtu.edu.cn

YANPING CHEN

School of Mathematical Sciences

South China Normal University

Guangzhou 510631, P. R. China

E-mail address: yanpingchen@scnu.edu.cn

YUNQING HUANG

HunAN Key Laboratory FOR COMPUTATION AND

Simulation in Science And Engineering

Xiangtan University

Xiangtan 411105, P. R. China

E-mail address: huangyq@xtu.edu.cn 